\title{
The role of particle-particle interactions in bubbling gas-fluidized beds of Geldart A particles: A discrete particle study
}

\author{
Junwu Wang', M.A. van der Hoef', J.A.M. Kuipers ${ }^{3}$ \\ Faculty of Science and Technology, University of Twente, P.O. Box 217, 7500 AE Enschede, The Netherlands. \\ E-mails: 1) J.Wang@tnw.utwente.nl, 2) M.A.vanderHoef@tnw.utwente.nl, 3) J.A.M.Kuipers@tnw.utwente.nl
}

\begin{abstract}
Discrete particle simulations are by now well established as an effective tool to study the mechanics of complex gas-solid flows in gas-fluidized beds. In this study, a state-of-the-art discrete particle model is used to explore the role of particle-particle interactions in bubbling gas-fluidized beds of Geldart A particles. We find that the particle-particle interactions, including inelastic particleparticle collision; inter-particle friction and slightly cohesive forces, only have a negligible effect on the hydrodynamics of bubbling gas-fluidized beds of Geldart A particles. This is due to the fact that only a very small fraction of the energy input is dissipated during the non-ideal particle-particle interaction. We finally show that the selected drag correlation model significantly affects the bed hydrodynamics.
\end{abstract}

Keywords: Geldart A particles; Fluidization; Multiphase flow; Discrete particle simulation; Particleparticle interaction; Bubbling fluidized beds

\section{Introduction}

Bubbling fluidized beds (BFBs) are widely used in the petroleum, energy and chemical industries. Correct understanding of the hydrodynamic characteristics of BFBs is essential for design and scale-up of such reactors. However, the analysis of such system is very difficult, because of the coexistence of disparate length and time scales and the conspicuous coupling between the different scales with their transport properties ( $\mathrm{Li}$ and Kwauk, 2003; van der Hoef et al., 2006). In order to obtain a fundamental insight into the complex hydrodynamics of BFBs, it is helpful to study the system from the basic interactions, i.e., the particle-particle interaction and the gas-particle interaction.

In recent decades, computer simulation has emerged as an effective tool to study the hydrodynamics of gas-fluidized beds (Gidaspow et al., 2004; Li et al., 2005; van der Hoef et al., 2008). Both Eulerian-Eulerian two-fluid model (TFM) and Eulerian-Lagrangian discrete particle model (DPM) are used to study the effect of particle-particle and gas-particle interactions on the hydrodynamics of BFBs, however, most of them are devoted to Geldart B and D particles (for example, Goldschmidt et al., 2002; Goldschmidt et al., 2001; Hoomans et al., 1996; $\mathrm{Li}$ and Kuipers, 2003, 2007; Lu et al., 2005; Reuge et al., 2008; Wang et al., 2008; Xu et al., 2007). Those studies concluded that particleparticle interaction significantly affected the bed hydrodynamics. For example, based on two-fluid modeling with kinetic theory of granular flows (KTGF) for particulate phase stresses, Goldschmidt et al. (2001) showed that when the restitution coefficient equals one (i.e. ideal particles), no bubbling state is obtained for a fluidization velocity above three times the minimum bubbling velocity (which is equal to the minimum fluidization velocity for such a particle), whereas when a physically correct restitution coefficient is used, the bed hydrodynamics are predicted reasonably well by the TFM.

It is well-known that particle properties have a significant effect on the bed hydrodynamics (Geldart, 1973). Therefore, it is interesting to study what will happen when Geldart A particles are used as the bed material. Kobayashi et al. 
(2002), Ye et al. (2004), Pandit et al. (2005; 2006) studied the effect of inter-particle cohesive forces on the homogeneous fluidization and the onset of minimum bubbling fluidization. However, only few studies are devoted to the hydrodynamics of Geldart A particles in BFBs, where all of them are based on TFM simulations. From those studies, a completely different picture as compared to B- and D-type particles emerged, namely, the restitution coefficient has no effect on the bubbling characteristics (Zimmermann and Taghipour, 2005), bed expansion characteristics (Wang et al., 2009b), minimum bubbling velocity and axial solid volume fraction profiles (Wang et al., 2009a). In these studies, the KTGF was used to represent the particle-particle interaction, which does include the effect of dissipation in collisions, but not the effect of particle-particle friction and/or inter-particle cohesive forces. So it remains as yet unclear how the latter two interactions will affect the bed hydrodynamics.

In this study, a state-of-the-art discrete particle model is used to explore the role of particle-particle interactions in BFBs of Geldart A particles. We show that the particle-particle normal restitution coefficient has a negligible effect on bed hydrodynamics, which confirm the results obtained from KTGF, we further find that the effect of particle-particle friction and slightly inter-particle cohesive forces are also negligible.

\section{Discrete particle model}

The DPM used here, which is pioneered by Tsuji et al. (1993) and Xu and Yu (1997), is part of the general multi-scale modeling strategy for numerical simulation of dense gas-solid fluidized beds (van der Hoef et al., 2008). The model has originally been developed by Ye et al. (2004), and is based on Hoomans et al. (1996). Below a summary of the main equations is given, details of the model can be found in Ye (2005) and van der Hoef et al. (2006).

In the DPM, the Eulerian grid size is usually far larger than the size of particles, so volume- averaged Navier-Stokes equations are used to describe the motion of the gas phase. The mass conservation equation is given by

$$
\frac{\partial}{\partial t}\left(\varepsilon_{g} \rho_{g}\right)+\nabla \cdot\left(\varepsilon_{g} \rho_{g} \mathrm{u}_{g}\right)=0
$$

where $\varepsilon_{g}, \rho_{g}$ and $\mathrm{u}_{g}$ represent the local porosity, gas density and gas velocity vector, respectively. The momentum conservation equation is given by

$$
\begin{aligned}
& \frac{\partial}{\partial t}\left(\varepsilon_{g} \rho_{g} \mathrm{u}_{g}\right)+\nabla \cdot\left(\varepsilon_{g} \rho_{g} \mathrm{u}_{g} \mathrm{u}_{g}\right) \\
& =-\varepsilon_{g} \nabla p-\mathrm{S}_{p}+\nabla \cdot\left(\varepsilon_{g} \overline{\bar{\tau}}_{g}\right)+\varepsilon_{g} \rho_{g} \mathrm{~g}
\end{aligned}
$$

where $p, \mathrm{~S}_{p}, \overline{\bar{\tau}}_{g}$ and $\mathrm{g}$ are pressure, source term, shear stress tensor and gravitational acceleration, respectively, and $\overline{\bar{\tau}}_{g}$ is calculated from

$$
\overline{\bar{\tau}}_{g}=\mu_{g}\left(\nabla \mathrm{u}_{g}+\left(\nabla \mathrm{u}_{g}\right)^{T}\right)-\frac{2}{3} \mu_{g}\left(\nabla \cdot \mathrm{u}_{g}\right) \overline{\bar{I}}
$$

where $\mu_{g}$ and $\overline{\bar{I}}$ are shear viscosity of gas phase and unit tensor, respectively, and $\rho_{g}$ is related to $p$ through equation of state of an ideal gas

$$
\rho_{g}=\frac{M_{g}}{R T_{g}} p
$$

where $R$ is the universal gas constant, $T_{g}$ is the temperature and $M_{g}$ is the molar mass of gas. The source term $\mathrm{S}_{p}$ in Eq.2 is defined as

$$
\mathrm{S}_{p}=\frac{1}{V_{\text {cell }}} \sum_{\forall a \in \text { cell }} \frac{\beta V_{a}}{1-\varepsilon_{g}}\left(\mathrm{u}_{g}-\mathrm{v}_{a}\right) \delta\left(r-r_{a}\right)
$$

where $V_{\text {cell }}$ represents the volume of a computational cell, $V_{a}$ the volume of particle $a$ and $\mathrm{v}_{a}$ the linear velocity of particle $a$. The $\delta$ function ensures that the drag force acts as a point force at the central position of this particle. $\beta$ is the inter-phase drag coefficient and is calculated by using the combined Ergun and Wen \& Yu correlation (Gidaspow, 1994)

$$
\beta_{\text {Gidaspow }}= \begin{cases}\frac{3}{4} C_{D} \frac{\rho_{g} \varepsilon_{g} \varepsilon_{p}\left|\mathrm{u}_{g}-\mathrm{v}_{a}\right|}{d_{p}} \varepsilon_{g}^{-2.65} & \varepsilon_{p} \leq 0.2 \\ 150 \frac{\varepsilon_{p}^{2} \mu_{g}}{\varepsilon_{g} d_{p}^{2}}+1.75 \frac{\rho_{g} \varepsilon_{p}\left|\mathrm{u}_{g}-\mathrm{v}_{a}\right|}{d_{p}} \varepsilon_{p}>0.2\end{cases}
$$


where $\varepsilon_{p}=1-\varepsilon_{g}$ and $d_{p}$ are solid volume fraction and particle diameter, respectively, and $C_{D}$ is calculated from the Schiller \& Naumann expression,

$$
\begin{gathered}
C_{D}=\left\{\begin{array}{ll}
\frac{24}{\operatorname{Re}_{p}}\left(1+0.15 \mathrm{Re}_{p}^{0.687}\right) & \operatorname{Re}_{p}<1000 \\
0.44, & \operatorname{Re}_{p} \geq 1000
\end{array},\right. \\
\operatorname{Re}_{p}=\frac{\varepsilon_{g} \rho_{g} d_{p}\left|\mathrm{u}_{g}-\mathrm{v}_{a}\right|}{\mu_{g}}
\end{gathered}
$$

Note that in DPM, the porosity is calculated by

$$
\varepsilon_{g}=1-\frac{1}{V_{\text {cell }}} \sum_{\forall a \in \text { cell }} f_{a} V_{a}
$$

where $f_{a} V_{a}$ is the fractional volume of particle $a$ residing in the cell under consideration.

The particle phase is described by the Newton equations of motion for each particle in the system. The translational and rotational equations of motion for a single particle $a$ are given by

$$
\begin{gathered}
m_{a} \frac{d \mathrm{v}_{a}}{d t}=-V_{a} \nabla p+\frac{V_{p} \beta}{1-\varepsilon_{g}}\left(\mathrm{u}_{g}-\mathrm{v}_{a}\right)+m_{a} \mathrm{~g}+\mathrm{F}_{c, a}+\mathrm{F}_{v d v, a} \\
I_{a} \frac{d \omega_{a}}{d t}=\mathrm{T}_{a}(10)
\end{gathered}
$$

where $m_{a}, I_{a}, \omega_{a}, \mathrm{~T}_{a}$ and $t$ represent particle mass, moment of inertia, angular velocity, torque and time, respectively. The first and second terms in the right hand side of Eq.9 originate from inter-phase interaction. The third term is caused by gravity, the fourth term is the contact force resulting from particle-particle or particlewall interaction, which is calculated using the soft-sphere model proposed by Cundall and Strack (1979). The fifth term is the inter-particle cohesive force, which is represented by using the van der Waals force $\left(\mathrm{F}_{v d w, a}\right)$, and is given by

$$
\mathrm{F}_{v d w, a}=\sum_{\forall b \in \text { Neighbourlist }} F_{v d w, a b} \mathrm{n}_{a b}
$$

$$
\begin{aligned}
& F_{v d w, a b}=-\frac{A}{3} \frac{2 r_{a} r_{b}\left(S+r_{a}+r_{b}\right)}{\left[S\left(S+2 r_{a}+2 r_{b}\right)\right]^{2}} \\
& \times\left[\frac{S\left(S+2 r_{a}+2 r_{b}\right)}{\left(S+r_{a}+r_{b}\right)^{2}-\left(r_{a}-r_{b}\right)^{2}}-1\right]^{2}
\end{aligned}
$$

where $\mathbf{n}_{a b}$ is the normal unit vector, $\mathrm{S}$ is the intersurface distance between two spheres, $A$ is the Hamaker constant, and $r_{a}$ and $r_{b}$ are the radii of the two spheres. Note in our case $r_{a}=r_{b}$. To avoid the singularity when the distance between two particles approaches zero, we define a maximal value of the van der Waals force between two particles, which is realized by using a cut-off value for the inter-surface distance, $S_{0}$.

In the numerical simulations, boundary and initial conditions have to be specified. At the top outlet, the pressure is specified as the atmospheric pressure (101325Pa), whereas at the bottom inlet, a uniform gas velocity profile with specific superficial gas velocity $\left(U_{g}\right)$ is imposed, free-slip wall is applied for gas phase as in a previous study (Ye et al., 2005). The particles are initially packed at the bottom of the bed with zero average velocity plus a random fluctuating velocity. Note that in our simulations, the tangential restitution coefficient is always set equal to the normal restitution coefficient and the parameters for the particle-wall interaction are equal to the parameters for the particle-particle interaction.

\section{Result and discussion}

As in the available studies on two-fluid modeling of Geldart A particles in BFBs, we have focused on the effect of particle-particle interaction. Table. 1 summarizes the parameters used in the simulations, which are similar to those in the study by Ye et al. (2005). Note that all of the simulations last 5s, the first second of which is used to equilibrate the system, and the remaining $4 \mathrm{~s}$ (which are free of start-up effects) are used to measure the various quantities of 
interest, which are sampled with a frequency of $10000 \mathrm{~Hz}$.

Table.1 A summary of the parameters used in the simulations

\begin{tabular}{|c|c|}
\hline Parameters & Value \\
\hline Particle number, $N_{\text {part }}$ & 36000 \\
\hline Particle diameter, $d_{p}$ & $7.5 \times 10^{-5} \mathrm{~m}$ \\
\hline Particle density, $\rho_{p}$ & $1495 \mathrm{~kg} / \mathrm{m}^{3}$ \\
\hline $\begin{array}{l}\text { Restitution coefficient, } \\
e_{\mathrm{n}}, e_{\mathrm{t}}\end{array}$ & $0.6,0.7,0.8,0.9,1.0$ \\
\hline Friction coefficient, $\mu_{f}$ & $0.1,0.2,0.3,0.4,0.5$ \\
\hline $\begin{array}{l}\text { Normal spring stiffness, } \\
k_{n}\end{array}$ & $7 \mathrm{~N} / \mathrm{m}$ \\
\hline 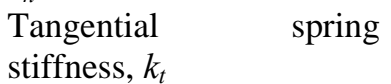 & $2 \mathrm{~N} / \mathrm{m}$ \\
\hline CFD time step & $1.0 \times 10^{-5} \mathrm{~s}$ \\
\hline $\begin{array}{l}\text { Particle dynamics time } \\
\text { step }\end{array}$ & $1.0 \times 10^{-6} \mathrm{~s}$ \\
\hline $\begin{array}{l}\text { Minimum inter-particle } \\
\text { distance, } S_{0}\end{array}$ & $0.4 \mathrm{~nm}$ \\
\hline Reactor height & $1.2 \times 10^{-2} \mathrm{~m}$ \\
\hline Reactor width & $3.0 \times 10^{-3} \mathrm{~m}$ \\
\hline Reactor depth & $1.2 \times 10^{-3} \mathrm{~m}$ \\
\hline Number of CFD cells & $48 \times 12 \times 5$ \\
\hline Gas temperature, $T_{g}$ & 293K \\
\hline Gas constant, $R$ & 8.314J/(mol·K) \\
\hline Molar mass of gas, $M_{g}$ & $28.8 \times 10^{-3} \mathrm{~kg} / \mathrm{mol}$ \\
\hline Shear viscosity of gas, $\mu_{g}$ & $1.8 \times 10^{-5}$ Pa.s \\
\hline
\end{tabular}

Snapshots from a simulation of a typical bubbling fluidized bed $\left(U_{g}=0.04 \mathrm{~m} / \mathrm{s}\right)$ are shown in Fig.1. There is no effect for different values of restitution coefficient $(e)$, friction coefficient $\left(\mu_{f}\right)$ and Hamaker constant ( $A$ ). From fig.1 (a), it can be seen that the normal particle-particle interaction does not affect the bubble characteristics, and even in case of $e=1$, bubbles can still be clearly detected, which is in agreement with previous simulation results for Geldart A particles using a two-fluid modeling (Wang et al., 2009a; Zimmermann and Taghipour, 2005), and is clearly different from the DPM results for coarse particles, where it was shown that particle collision properties have a profound effect on bed hydrodynamics, where in case of $e=1$, no two-phase structure emerges at all (Li and Kuipers, 2007).
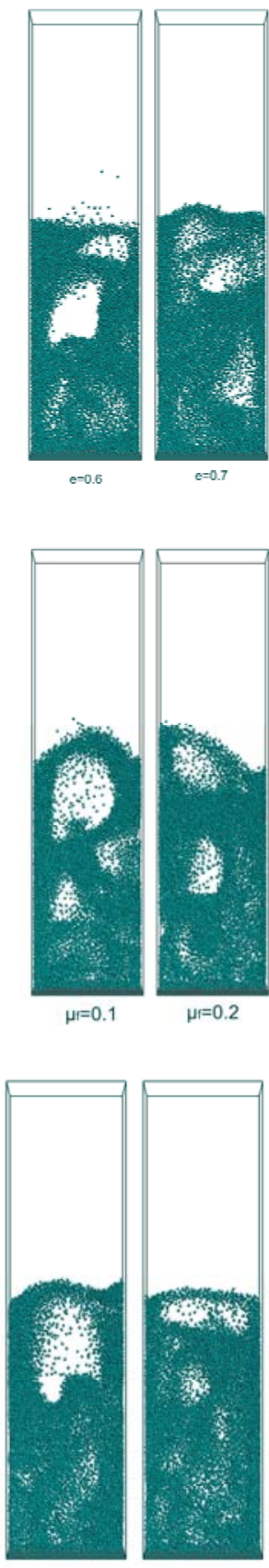

$A=1.0 \times 10^{22} \mathrm{~J}$

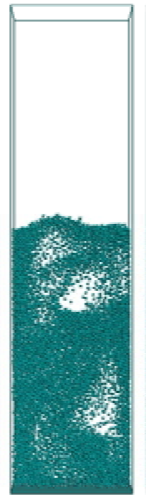

$\mathrm{e}=0.7$

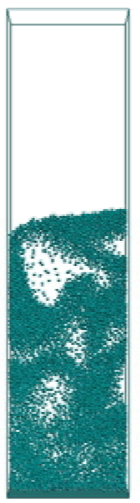

(a)

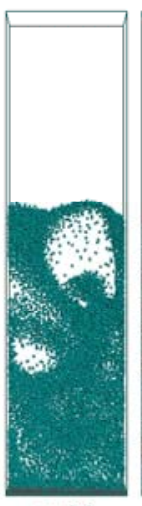

(b)

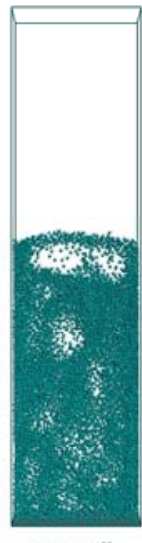

$A=5.0 \times 10^{22} \mathrm{~J}$

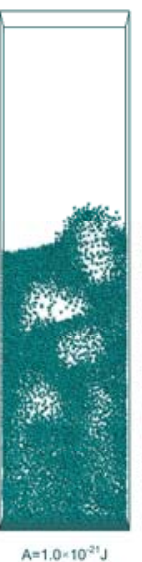

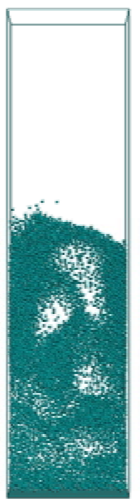

$e=0.9$

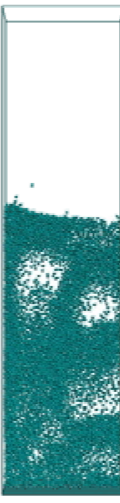

$=10$

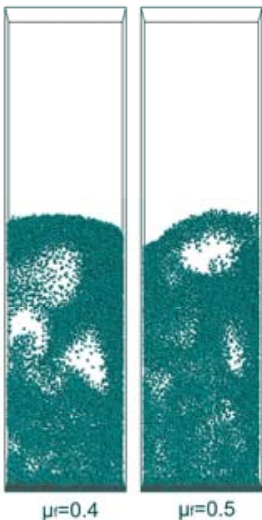

$\mu=0.5$

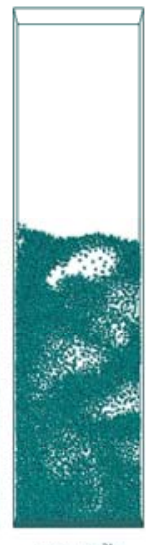

$A=5.0 \times 10^{21} \mathrm{~J}$

(c)
Figure.1 Snapshots of simulation results taken at a slit (the particles located at the dimensionless bed depth between 0.35 and 0.65 are shown). Showing the effect of restitution coefficient (a), friction coefficient (b) and Hamaker constant (c). $\mathrm{U}_{\mathrm{g}}=0.04 \mathrm{~m} / \mathrm{s}$. In (a) the interparticle friction and cohesive forces are not included; in (b) $e=0.9$, the effect of cohesive force is not included; in (c) $e=0.9, \mu_{f}=0.3$. 
By comparing fig.1 (a) with fig.1 (b), we conclude that the inclusion of the effect of interparticle friction has no visible effect on the bed structures, which is again in strong contrast with the observations for coarse particles ( $\mathrm{Li}$ and Kuipers, 2007). Fig.1 (c) indicates that within the ranges we studied, the effect of cohesive forces are negligible, which can be understood from the fact that the cohesive force is a short range interaction. Since the average porosity in our cases is about 0.6-0.7, the mean inter-particle distance is large, so the effect of van der Waals forces becomes extremely weak. However, it must be pointed out that the conclusion obtained here is somewhat different from one of our previous 2D DPM studies on Geldart A particles (Ye et al., 2004), where it was shown that when $\mathrm{A}=10^{-20} \mathrm{~J}$, chain-like structures are formed and the inter-particle cohesive force is the dominant source of the velocity fluctuation of particles.

A possible origin could be that the interparticle cohesive force not only depends on the Hamaker constant but also on the inter-particle distance, and that for the same solid volume fraction and Hamaker constant, the inter-particle distance in $2 \mathrm{D}$ is less than in $3 \mathrm{D}$, which means that the inter-particle cohesive force in $3 \mathrm{D}$ is weaker than the one in $2 \mathrm{D}$. We can image that with decreasing $U_{g}$, the cohesive force will play an increasing role in the fluidization of Geldart A particles, however, we do not address this issue here, because (1) a large number of DPM simulations have already been devoted to homogeneous fluidization and the onset of minimum bubbling fluidization as stated in the introduction section; (2) the cohesive force used here hardly represents the true nature of interparticle cohesive forces; and most importantly, (3) industrial gas-fluidized beds are seldom operated at such low superficial gas velocity.

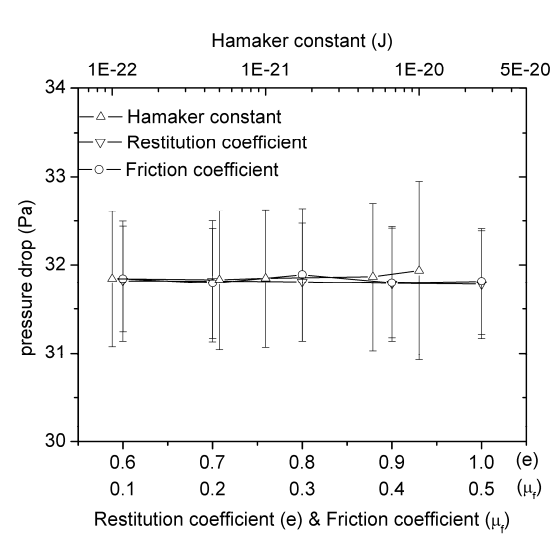

(a)

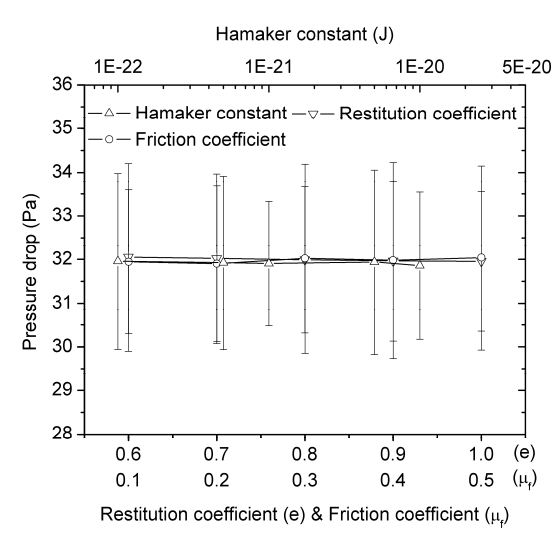

(b)

Figure.2 The effect of particle-particle interaction on the pressure drop and its deviation, (a) $U_{g}=0.02 \mathrm{~m} / \mathrm{s}$; (b) $U_{g}=0.04 \mathrm{~m} / \mathrm{s}$

Fig.2 shows the effect of particle-particle interaction on the pressure drop at $U_{g}=0.02 \mathrm{~m} / \mathrm{s}$ and $U_{g}=0.04 \mathrm{~m} / \mathrm{s}$, respectively. It can be seen that particle-particle interaction does not affect the pressure drop and its standard deviation, which is again in contrast with the observations for coarse particles as reported by $\mathrm{Li}$ and Kuipers (2007), where it was shown that due to the significant change in the spatial and temporal structures caused by different particle-particle interaction characteristics, stronger collisional dissipation leads to stronger pressure oscillations.

Also for the time-average particle height, the effect of the particle interaction is marginal as shown in fig.3, where the particle height is defined as (Goldschmidt et al., 2004) 


$$
\left\langle h_{p}\right\rangle_{\text {bed }}=\frac{\sum_{i=1}^{N_{\text {part }}} h_{i}}{N_{\text {part }}}
$$

It worthwhile to mention that without the inclusion of particle friction and cohesive forces, a higher restitution coefficient results in higher time-average particle height. In case of a superficial gas velocity of $0.04 \mathrm{~m} / \mathrm{s}$, the timeaverage particle height with $e=1$ is about $6 \%$ higher than the value obtained by using $e=0.6$. Since this variation is within the reported standard deviations of time-average particle height, we still conclude that particle-particle interaction has no pronounced effect on the timeaverage particle height.

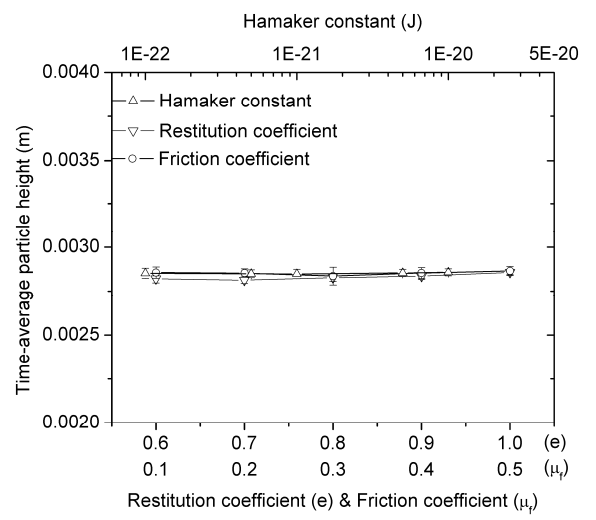

(a)

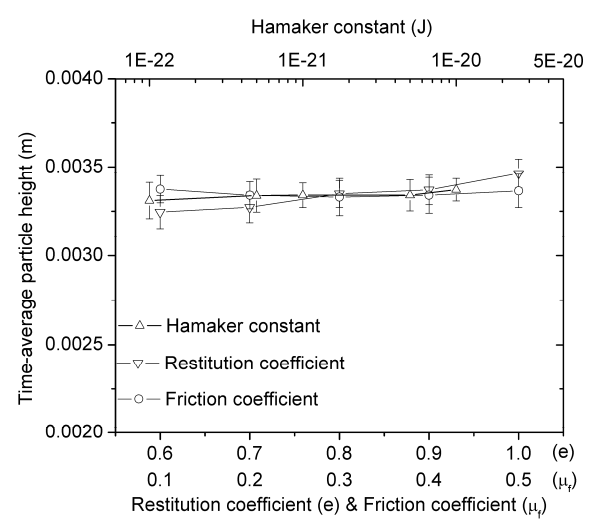

(b)

Figure.3 The effect of particle-particle interaction on the time-average particle height, (a) $U_{g}=0.02 \mathrm{~m} / \mathrm{s}$; (b) $U_{g}=0.04 \mathrm{~m} / \mathrm{s}$
Fig.4 shows the effect of particle-particle interaction on the global granular temperature, which is defined as,

$$
\Theta=\frac{1}{3}\left(\Theta_{x}+\Theta_{y}+\Theta_{z}\right)
$$

where the granular temperature in the $k$-direction $\Theta_{k}$, is defined as

$$
\Theta_{k}=\frac{\sum_{i=1}^{N_{\text {part }}}\left(v_{k, i}-\bar{v}_{k}\right)^{2}}{N_{\text {part }}}
$$

and the average velocity $\bar{v}_{k}$, is defined as

$$
\bar{v}_{k}=\frac{\sum_{i=1}^{N_{\text {part }}} v_{k, i}}{N_{\text {part }}}
$$

Note that this definition of granular temperature indicates that the effect of heterogeneous structures on the granular temperature is already included. It can be seen that all computed granular temperatures for same $U_{g}$ are comparable. Since in BFBs, the granular temperature is dominated by the bubble structure (Campbell and Wang, 1991; Menon and Durian, 1997), this means that the bubble structure is not affected by the particle-particle interaction. Based on our results, we therefore conclude that for Geldart A particles, particle-particle interaction only plays a negligible role in the structure formation of BFBs.

It is as yet unclear why the role played by particle-particle interaction is negligible for Geldart A particles. Recently, a series of studies (Goldschmidt et al., 2004; Li and Kuipers, 2002, 2004, 2007) have revealed that energy budget analysis is a powerful tool to analyze the underlying mechanics of gas-solid flows. In Fig.5, we show the effect of restitution coefficient on the energy budget analysis at a superficial gas velocity of $0.04 \mathrm{~m} / \mathrm{s}$ without the presence of friction and cohesive forces. In fig.5, $f_{k i n}, f_{\text {pot }}$, $f_{\text {sprn }}$ and $f_{\text {dispn }}$ represnt the fraction of kinetic energy, potential energy, potential energy of the normal spring and energy dissipation by the normal spring of the total particle energy, respectively. 


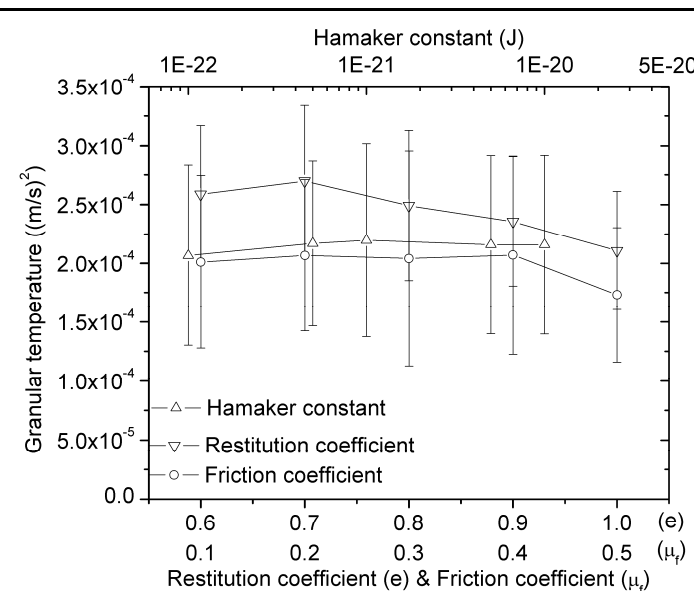

(a)

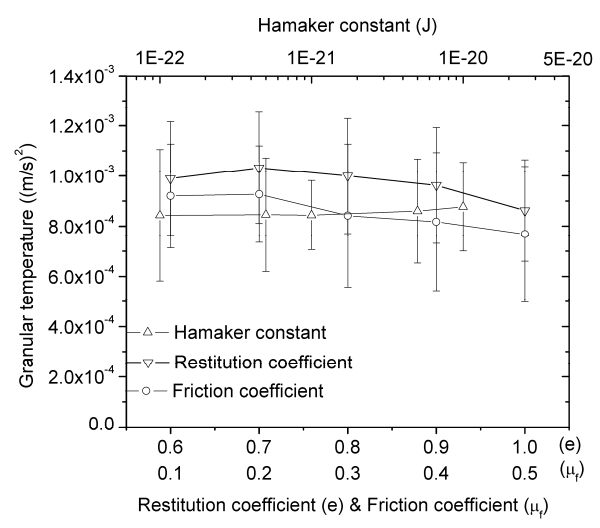

(b)

Figure.4 The effect of particle-particle interaction on the global granular temperature, (a) $U_{g}=0.02 \mathrm{~m} / \mathrm{s}$; (b) $U_{g}=0.04 \mathrm{~m} / \mathrm{s}$

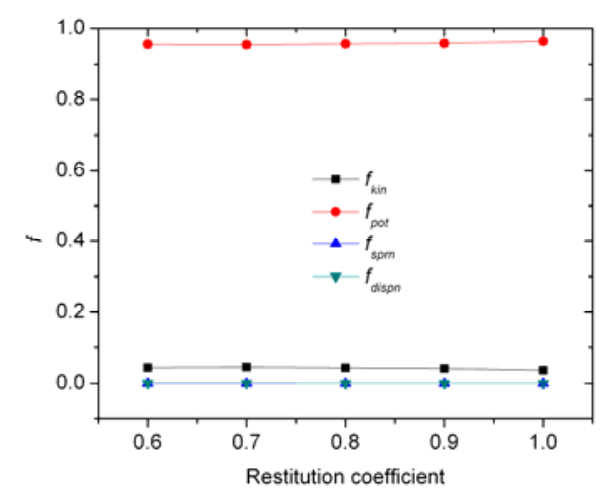

Figure.5 Energy budget analysis of particle phase, $U_{g}=0.04 \mathrm{~m} / \mathrm{s}$, without consideration of the effect of interparticle friction and cohesive force.

Details of their mathematical expressions can be found in Ye (2005). Note that the results for all cases are similar, therefore, for simplicity only the effect of restitution coefficient is shown. The energy budget analysis on the particle phase reveals that almost all of the energy input is used to keep the particles in suspension with a constant granular temperature (i.e. kinetic energy), only a very small fraction of the energy input (less than $1 \%$ in all cases we studied) is dissipated in the non-ideal particle-particle interactions.

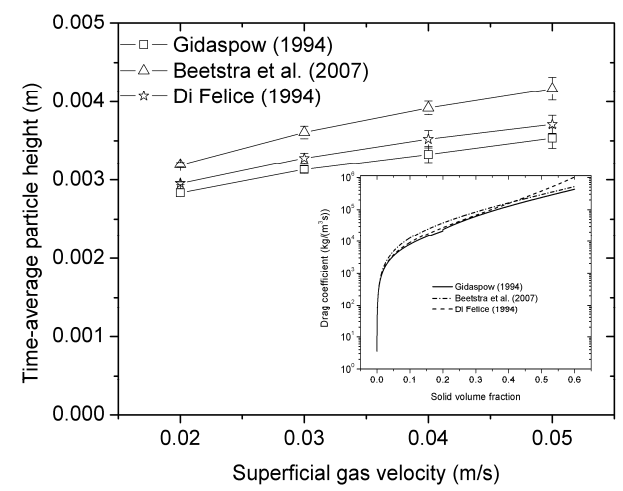

Figure.6 The effect of drag correlations on the timeaverage particle height, the inset shows the comparison of the drag coefficients by Ergun and Wen and $\mathrm{Yu}$ (Gidaspow, 1994), Di Felice (1994) and Beetstra et al. (2007) at an inter-phase slip velocity of $0.04 \mathrm{~m} / \mathrm{s}$.

Finally, simulations are performed in order to study the effect of drag correlations. Two different drag correlations are considered, one which is obtained using lattice Boltzmann simulations (Beetstra et al., 2007),

$$
\begin{gathered}
\beta_{\text {Beetstra }}=180 \frac{\mu_{g} \varepsilon_{p}^{2}}{d_{p}^{2} \varepsilon_{g}}+18 \frac{\mu_{g} \varepsilon_{g}^{3} \varepsilon_{p}\left(1+1.5 \sqrt{\varepsilon_{p}}\right)}{d_{p}^{2}} \\
+0.31 \frac{\mu_{g} \varepsilon_{p} \operatorname{Re}_{p}}{\varepsilon_{g} d_{p}^{2}} \frac{\left[\varepsilon_{g}^{-1}+3 \varepsilon_{g} \varepsilon_{p}+8.4 \operatorname{Re}_{p}^{-0.343}\right]}{\left[1+10^{3 \varepsilon_{p}} \operatorname{Re}_{p}^{-0.5-2 \varepsilon_{p}}\right]}
\end{gathered}
$$

and the other is developed by Di Felice (1994) which is widely used in DPM community:

$$
\beta_{\text {Felice }}=\frac{3}{4} C_{D} \frac{\rho_{g} \varepsilon_{p}\left|\mathrm{u}_{g}-\mathrm{v}_{a}\right|}{d_{p}} \varepsilon_{g}^{-\chi}
$$




$$
\begin{gathered}
C_{D}=\left(0.63+\frac{4.8}{\sqrt{\mathrm{Re}_{p}}}\right)^{2}, \\
\chi=2.7-0.65 \exp \left[-\frac{\left(1.5-\log _{10} \mathrm{Re}_{p}\right)^{2}}{2}\right]
\end{gathered}
$$

Fig.6 shows the effect of drag correlations on the time-average particle height with $\mathrm{e}=0.9$ and $\mu_{f}$ $=0.3$. It can be seen that the drag correlation has a profound effect on the time-average particle height. For example, for the case of a superficial gas velocity of $0.05 \mathrm{~m} / \mathrm{s}$, the value simulated using the drag correlation of Beetstra et al. (2007) is about $18 \%$ higher than the one obtained using Gidaspow's correlation (1994). Even for a lower superficial gas velocity of $0.02 \mathrm{~m} / \mathrm{s}$, the relative error is still larger than $12 \%$. This is obviously due to the fact that the drag coefficient of Beetstra et al. (2007) is larger than the one by Gidaspow (1994) as shown in the inset of fig.6. However, because no corresponding experimental data is available, it is unclear which correlation is best for the simulation of Geldart A particles in BFBs. It should be born in mind that due to the limitation of computational resources, only a very small system can be simulated by DPM, so that, special experimental studies of micro-gas-fluidized beds are necessary to assess which correlation is the best.

\section{Conclusion}

A state-of-the-art discrete particle model is used to explore the role of particle-particle interaction in bubbling gas-fluidized beds of Geldart A particles. We find that in general particle-particle inelastic interactions do not affect the typical bed characteristics that we studied, such as the pressure drop and its standard deviation, the time-average particle height and the global granular temperature. This confirms the previous conclusion based on the two-fluid modeling of Geldart A particles with kinetic theory of granular flows for particulate phase stresses. We further show that the presences of particle-particle friction and slightly cohesive forces also have a negligible effect, from which we conclude that particle-particle interaction only plays a negligible role in the hydrodynamics of bubbling gas-fluidized beds of Geldart A particles. Finally, we showed that the inter-phase drag correlation used, which reflects the gas-particle interaction in the DPM simulations, constitutes a control factor in the bed hydrodynamics.

\section{Acknowledgement}

This research project is part of the research program of the "Towards a reliable model for industrial gas-fluidized bed reactors with polydisperse", which is financially supported by NWO.

\section{References}

Beetstra R, van der Hoef MA, Kuipers JAM. 2007. Drag force of intermediate Reynolds number flow past mono- and bidisperse arrays of spheres. A.I.Ch.E Journal 53, 489-501.

Campbell CS, Wang DA. 1991. Particle pressures in gasfluidized beds. Journal of Fluid Mechanics 227, 495508.

Cundall PA, Strack ODL. 1979. A discrete numerical model for granular assemblies. Geotechniques 29, 4765.

Di Felice R. 1994. The voidage function for fluid-particle interaction systems. International Journal of Multiphase Flow 20, 153-159.

Geldart D. 1973. Types of gas fluidization. Powder Technology 7, 285-292.

Gidaspow D. 1994. Multiphase flow and fluidization: continuum and kinetic theory description. Academic Press

Gidaspow D, Jung J, Singh RK. 2004. Hydrodynamics of fluidization using kinetic theory: an emerging paradigm. Powder Technology 148 123-141.

Goldschmidt MJV, Beetstra R, Kuipers JAM. 2002. Hydrodynamic modelling of dense gas-fluidised beds: Comparison of the kinetic theory of granular flow with 3D hard-sphere discrete particle simulations. Chemical Engineering Science 57, 2059-2075.

Goldschmidt MJV, Beetstra R, Kuipers JAM. 2004. Hydrodynamic modelling of dense gas-fluidised beds: comparison and validation of 3D discrete particle and continuum models. Powder Technology 142, 23-47. 
Goldschmidt MJV, Kuipers JAM, van Swaaij WPM. 2001. Hydrodynamic modelling of dense gas-fluidised beds using the kinetic theory of granular flow: effect of coefficient of restitution on bed dynamics. Chemical Engineering Science 56, 571-578.

Hoomans BPB, Kuipers JAM, Briels WJ, van Swaaij WPM. 1996. Discrete particle simulation of bubble and slug formation in a two-dimensional gas-fluidised bed: A hard-sphere approach. Chemical Engineering Science 51, 99-108.

Kobayashi T, Kawaguchi T, Tanaka T, Tsuji Y. 2002. DEM analysis on flow pattern of Geldart's group A particles in fluidized bed. Proceedings of the World Congress on Particle Technology 4 (CD-ROM), July 21-25, Sydney, Australia, Paper no. 178.

Li J, Kuipers JAM. 2002. Effect of pressure on gas-solid flow behavior in dense gas-fluidized beds: a discrete particle simulation study. Powder Technology 127, 173-184.

Li J, Kuipers JAM. 2003. Gas-particle interaction in dense gas-fluidized beds. Chemical Engineering Science 58, 711-718.

Li J, Kuipers JAM. 2004. Flow structure formation and evolution in circulating gas-fluidized beds. China Particuology 2, 1-12.

Li J, Kuipers JAM. 2007. Effect of competition between particle-particle and gas-particle interactions on flow patterns in dense gas-fluidized beds. Chemical Engineering Science 62, 3429-3442.

Li J, Kwauk M. 2003. Exploring complex systems in chemical engineering: the multi-scale methodology. Chemical Engineering Science 58, 521-535.

Li J, Ouyang J, Gao S, Ge W. 2005. Multi-scale simulation of particle-fluid complex systems. Science Press. Beijing, China.

Lu H, Wang S, Zhao Y, Gidaspow D. 2005. Prediction of particle motion in a two-dimensional bubbling fluidized bed using discrete hard-sphere model. Chemical Engineering Science 60, 3217-3231.

Menon N, Durian DJ. 1997. Particle motion in gasfluidized bed of sand. Physical Review Letters 79, 3407-3410.

Pandit JK, Wang XS, Rhodes MJ. 2005. Study of Geldart's Group A behaviour using the discrete element method simulation. Powder Technology 160, 7-14.

Pandit JK, Wang XS, Rhodes MJ. 2006. On Geldart Group A behaviour in fluidized beds with and without cohesive interparticle forces: A DEM study. Powder Technology 164, 130-138.

Reuge N, Cadoret L, Coufort-Saudejaud C, Pannala S, Syamlal M, Caussat B. 2008. Multifluid Eulerian modeling of dense gas-solids fluidized bed hydrodynamics: Influence of the dissipation parameters. Chemical Engineering Science 63, 5540-5551.

Tsuji Y, Kawaguchi T, Tanaka T. 1993. Discrete particle simulation of two-dimensional fluidized bed. Powder Technology 77, 79-89.

van der Hoef MA, van Sint Annaland M, Deen NG, Kuipers JAM. 2008. Numerical simulation of dense gas-solid fluidized beds: A multiscale modeling strategy. Annual Review of Fluid Mechanics 40, 47-70.

van der Hoef MA, Ye $M$, van Sint Annaland $M$, Andrews.IV AT, Sundaresan S, Kuipers JAM. 2006. Multi-scale modeling of gas-fluidized beds. Advances in Chemical Engineering 31, 65-149.

Wang J, Van der Hoef MA, Kuipers JAM. 2009a. Twofluid modeling of minimum bubbling velocity of Geldart A particles in a gas-fluidized bed. submitted to Chemical Engineering Science

Wang J, Van der Hoef MA, Kuipers JAM. 2009b. Why the two-fluid model fails to predict the bed expansion characteristics of Geldart A particles in gas-fluidized beds: A tentative answer. Chemical Engineering Science 64, 622-625.

Wang S, Lu H, Li X, Yu L, Ding J, Zhao Y. 2008. CFD simulations of bubbling beds of rough spheres. Chemical Engineering Science 63, 5653-5662.

$\mathrm{Xu} \mathrm{BH}, \mathrm{Yu}$ AB. 1997. Numerical simulation of the gassolid flow in a fluidized bed by combining discrete particle method with computational fluid dynamics. Chemical Engineering Science 52, 2785-2809.

Xu M, Ge W, Li J. 2007. A discrete particle model for particle-fluid flows with considerations of sub-grid structures. Chemical Engineering Science 62, 2302 2308.

Ye M. 2005. Multi-level modeling of dense gas-solid twophase flows. PhD thesis. University of Twente, Enschede, Netherlands.

Ye M, van der Hoef MA, Kuipers JAM. 2004. A numerical study of fluidization behavior of Geldart A particles using a discrete particle model. Powder Technology 139, 129-139.

Ye M, van der Hoef MA, Kuipers JAM. 2005. The effects of particle and gas properties on the fluidization of Geldart A particles. Chemical Engineering Science 60, 4567-4580.

Zimmermann S, Taghipour F. 2005. CFD modeling of the hydrodynamics and reaction kinetics of FCC fluidizedbed reactors. Industrial \& Engineering Chemistry Research 44, 9818-9827. 